\title{
Some Bounds on Greatest Common Divisor Degree Estrada Index of Graphs
}

\author{
K. Nagarajan, R. S. Ramkumar
}

\begin{abstract}
Let $G$ be a simple graph of order $n$. In this paper we find some bounds on Greatest Common Divisor Degree Estrada Index of the graph $G$ by using mathematical inequalities interms of G.C.D. degree Index $I_{G C D}$ of graphs and exponential terms.
\end{abstract}

Index Terms - G.C.D. degree Energy, G.C.D. degree Estrada Index, G.C.D. degree spectral radius, G.C.D. degree Indexof graphs.

\section{INTRODUCTION}

In 1736 , the concept of graph theory was developed by Euler. Graph theory is applied in all fields for solving combinatorial problems in various areas such as operations research, optimization, number theory and computer science. I.G.Gutman introduced a Energy of graphs in 1978. Spectral theory is a potential area of interdisciplinary research and Energy of graph is of recent interest. We introduced a greatest common degree energy of graphs and discussed about the greatest common divisor degree energy of some standard graphs[10]. Here we find some bounds for the greatest common divisor degree Estrada Index of graphs.

\section{PRELIMINARIES}

Here, we see some definitions and theoremsconcerned with energy of graphs and greatest common divisor energy of graphs for proving the main results in this paper. $\left\{v_{1}, v_{2}, \ldots, v_{n}\right\}$ be its vertex set. The adjacency matrix $A(G)=\left[a_{i j}\right]$ of the graph $G$ is defined as a $n \times n$ matrix where $a_{i j}$ is 1 if the vertices $v_{i}$ and $v_{j}$ are adjacent or otherwise it is 0 .

Definition 2.2.[6] Let $G$ be a simple graph of order $n$ and consider the adjacency matrix $A(G)$ of the graph $G$. The eigen values $\lambda_{1}, \lambda_{2}, \ldots, \lambda_{n}$ of $A(G)$ where $\lambda_{1} \geq \lambda_{2} \geq \cdots \geq \lambda_{n}$ are the eigen values of the graph $G$. Then the energy $E(G)$ of $G$ is defined as add up the absolute values of its eigen values that is $E(G)=\sum_{i=1}^{n}\left|\lambda_{i}\right|$.

Definition 2.3. [10]Let $G$ be a simple graph and let $v_{1}, v_{2}, \ldots, v_{n}$ be its vertices .Let degree of $v_{i}=d_{i}$ for every $i=1,2, \ldots, n$. Define

$$
a_{i j}=\left\{\begin{array}{ll}
g . c . d & \left(d_{i}, d_{j}\right) \text { if } v_{i}, v_{j} \text { areadjacent } \\
0 & \text { otherwise }
\end{array} .\right.
$$

Then the square matrix $M(G)=\left[a_{i j}\right]$ of order $n$ is called a greatest common divisor degree matrix (G.C.D.

\footnotetext{
Revised Manuscript Received on December 15, 2019
}

K. Nagarajan *, Department of Mathematics, Kalasalingam University, Krishnankoil, Tamil Nadu, India. Email: k_nagarajan_srnmc@yahoo.co.in

R. S. Ramkumar, Department of Mathematics,Sri S.Ramasamy Naidu Memorial College, Sattur,Tamil Nadu, India. Email: nawin.urs@ gmail.com
Definition 2.1.[6] Let $G$ be a simple graph and let $V(G)=$

degree matrix) of $G$. The characteristic polynomial of the G.C.D. degree matrix $M(G)$, denoted by $\phi(G ; \lambda)$, is definedby $\quad \phi(G ; \lambda)=\operatorname{det}(\lambda I-M(G))=\lambda^{n}+$ $c_{1} \lambda^{(n-1)}+\ldots+c_{n}$ where I denote the $n \times$ nidentity matrix. The roots $\lambda_{1}, \lambda_{2}, \ldots, \lambda_{n}, \lambda_{1} \geq \lambda_{2} \geq \cdots \geq \lambda_{n}$, of the polynomial $\phi(G ; \lambda)$ are the greatest common divisor degree eigen values (G.C.D. degree eigen values) of $G$. The greatest common divisor degree energy (G.C.D. degree energy) of a graph $G$ is $E_{G C D}(G)=\sum_{i=1}^{n}\left|\lambda_{i}\right|$. Among the G.C.D. degree eigen values $\lambda_{1}, \lambda_{2}, \ldots, \lambda_{n}$, the largest G.C.D. degree eigen value is called a G.C.D. degree spectral radius of $G$.

Example 2.4.Consider a graph G. Here, $d\left(v_{1}\right)=3$,

$$
d\left(v_{2}\right)=1, d\left(v_{3}\right)=2, d\left(v_{4}\right)=2 \text {. }
$$

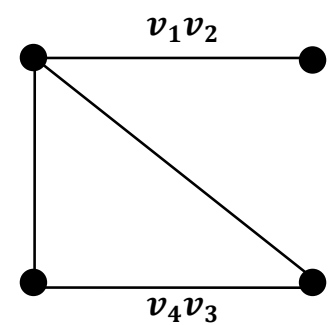

Fig 1

From fig.1, the G.C.D. degree matrix of the graph $G$ is $M(G)=\left(\begin{array}{llll}0 & 1 & 1 & 1 \\ 1 & 0 & 0 & 0 \\ 1 & 0 & 0 & 2 \\ 1 & 0 & 2 & 0\end{array}\right)$. Then the G.C.D. degree eigen values are $\lambda_{1}=-2, \lambda_{2} \approx-1.343, \lambda_{3} \approx 0.529$ and $\lambda_{4} \approx 2.814$ and the greatest common divisor degree energy of the graph $G$ is $E_{G C D}(G) \cong 6.686$.

Theorem 2.5. [9]Assume thatG is a simple graph with G.C.D. degree index $I_{G C D}$. Then, $\lambda_{1} \geq \frac{2 I_{G C D}}{n}$ and also $\lambda_{1}=\frac{2 I_{G C D}}{n}$ if and only if $G$ is $k-G . C . D$. degree regular.

Definition 2.6. [11]Assume that $G$ is a $(n, m)$-graphwhich is simple and connected. Then the G.C.D. degree Estrada index of the graph $E E_{G C D}(G)$ is defined by $E E_{G C D}(G)=$ $\sum_{\mathrm{i}=1}^{\mathrm{n}} \mathrm{e}^{\lambda_{\mathrm{i}}}$ where $\lambda_{1} \geq \lambda_{2} \geq \cdots \geq \lambda_{\mathrm{n}}$ are G.C.D. degree eigen values of $G$.

$$
\begin{gathered}
\text { Let } N_{t}=\sum_{i=1}^{n}\left(\lambda_{\mathrm{i}}\right)^{t} \\
\text { Then } E_{G C D}(G)=\sum_{\mathrm{t} \geq 0} \frac{N_{t}}{\mathrm{t} !} \\
N_{0}=\sum_{i=1}^{n}\left(\lambda_{\mathrm{i}}\right)^{0}=n
\end{gathered}
$$




\section{Some Bounds on Greatest Common Divisor Degree Estrada Index of Graphs}

$$
\begin{gathered}
N_{1}=\sum_{i=1}^{n}\left(\lambda_{\mathrm{i}}\right)^{1}=\text { trace of } M(G)=0 \\
N_{2}=\sum_{i=1}^{n}\left(\lambda_{\mathrm{i}}\right)^{2}=2\left[\sum_{1 \leq i<j \leq n}\left[\text { g.c.d. }\left(d_{i}, d_{j}\right)\right]^{2}\right]
\end{gathered}
$$

\section{BOUNDS ON GREATEST COMMON DIVISOR DEGREE ESTRADA INDEX OF GRAPHS}

Here we find some bounds on G.C.D. degree Estrada index of graphs.

Theorem 3.1Assume that $G$ is a $(n, m)$-graphwhich is simple and connected. Then for any integer $m_{0} \geq 2, E E_{G C D}(G) \geq$

$\sqrt{n^{2}+\sum_{t=2}^{m_{0}} \frac{2^{t} N_{t}(G)}{t !}}$ and $E E_{G C D}(G)$ becomes equal to its lower bound if and only if $G \cong K_{1}$.

Proof:

Now $N_{t}(G)$ is equal to $[M(G)]^{t}$ where $M(G)$ is the G.C.D. degree matrix of $\mathrm{G}$.

Thus $\sum_{t \geq m_{0}+1} \frac{2^{t} N_{t}(G)}{\mathrm{t} !} \geq 0$

$$
\text { Then } \begin{aligned}
\sum_{i=1}^{n} & e^{2 \lambda_{i}}=\sum_{i=1}^{n} \sum_{t \geq 0} \frac{\left(2 \lambda_{i}\right)^{t}}{t !} \\
& =\sum_{t \geq 0} \frac{2^{t} N_{t}(G)}{\mathrm{t} !} \\
& \geq \sum_{t=0}^{m_{0}} \frac{2^{t} N_{t}(G)}{\mathrm{t} !}
\end{aligned}
$$

Also, $2 \sum_{1 \leq i<j \leq n} e^{\lambda_{i}} e^{\lambda_{j}} \geq n(n-1)\left(\prod_{i<j} e^{\lambda_{i}} e^{\lambda_{j}}\right)^{\frac{2}{n(n-1)}}$

$$
\begin{aligned}
=n(n-1) & {\left[\left(\prod_{i=1}^{n} e^{\lambda_{i}}\right)^{n-1}\right]^{\frac{2}{n(n-1)}} } \\
= & n(n-1)\left(e^{N_{1}}\right)^{\frac{2}{n}} \\
= & n(n-1)
\end{aligned}
$$

Therefore, $\left[E E_{G C D}(G)\right]^{2}=\sum_{i=1}^{n} e^{2 \lambda_{\mathrm{i}}}+2 \sum_{1 \leq i<j \leq n} e^{\lambda_{i}} e^{\lambda_{j}}$

$$
\geq n(n-1)+\sum_{t=0}^{m_{0}} \frac{2^{t} N_{t}(G)}{\mathrm{t} !}
$$

$E E_{G C D}(G)=\sqrt{n^{2}+\sum_{t=2}^{m_{0}} \frac{2^{t} N_{t}(G)}{t !}}$ if and only if all the G.C.D. degree eigen values are 0 that is $G \cong K_{1}$.

Theorem 3.2 Let Assume that $G$ is a graphwith $n \geq 2$ verticeswhich is simple and connected and G.C.D. degree index $I_{G C D}$. Then $E E_{G C D}(G) \geq e^{\frac{2 I_{G C D}}{n}}+(n-1) e^{\frac{-2 I_{G C D}}{n(n-1)}}$ and $E E_{G C D}(G)$ becomes equal to its lower bound if and only if $G \cong K_{1}$.

Proof:

$$
\begin{gathered}
\text { Now, } E E_{G C D}(G)=e^{\lambda_{i}}+\sum_{i=2}^{n} e^{\lambda_{i}} \\
\geq e^{\lambda_{1}}+(n-1)\left(\prod_{i=2}^{n} e^{\lambda_{i}}\right)^{\frac{1}{n-1}} \\
=e^{\lambda_{1}}+(n-1) e^{\frac{\sum_{i=2}^{n} \lambda_{i}}{n-1}} \\
=e^{\lambda_{1}}+(n-1) e^{\frac{-\lambda_{1}}{n-1}} \\
\geq e^{\frac{2 I_{G C D}}{n}}+(n-1) e^{\frac{-2 I_{G C D}}{(n-1)}}
\end{gathered}
$$

$E E_{G C D}(G)=e^{\frac{2 I_{G C D}}{n}}+(n-1) e^{\frac{-2 I_{G C D}}{n(n-1)}}$ if and only if all the G.C.D. degree eigen values are 0 that is $G \cong K_{1}$.
Theorem 3.3Assume that $G$ is a $(n, m)$-graphwhich is simple and connected. Then $E E_{G C D}(G) \geq n-2 m$ and

$E E_{G C D}(G)=n-2 m$ if and only if $G \cong K_{1}$.

Proof:

Now, $E E_{G C D}(G)=\sum_{i=1}^{n} \sum_{t \geq 0} \frac{\left(\lambda_{i}\right)^{t}}{t !}$

$$
=n+2\left[\sum_{1 \leq i<j \leq n}\left[\text { g.c.d. }\left(d_{i}, d_{j}\right)\right]^{2}\right]+\sum_{i=1}^{n} \sum_{t \geq 2} \frac{\left(\lambda_{i}\right)^{t}}{t !}
$$

As $\mathrm{e}^{\lambda_{\mathrm{i}}} \geq 1+\lambda_{\mathrm{i}}$ holds for alli, $\sum_{t \geq 2} \frac{\left(\lambda_{i}\right)^{t}}{t !}$.

Let $\varepsilon \in[0,1]$.

Hence, $E E_{G C D}(G) \geq n+2\left[\sum_{1 \leq i<j \leq n}\left[\text { g.c.d. }\left(d_{i}, d_{j}\right)\right]^{2}\right]-$ $n \varepsilon-2 \varepsilon\left[\sum_{1 \leq i<j \leq n}\left[\text { g.c.d. }\left(d_{i}, d_{j}\right)\right]^{2}\right]+\varepsilon \sum_{i=1}^{n} \sum_{t \geq 0} \frac{\left(\lambda_{i}\right)^{t}}{t !}$

$$
\begin{gathered}
=(1-\varepsilon) n+2(\varepsilon-1)\left[\sum_{1 \leq i<j \leq n}\left[\text { g.c.d. }\left(d_{i}, d_{j}\right)\right]^{2}\right] \\
+E E_{G C D}(G)
\end{gathered}
$$

Therefore $E E_{G C D}(G) \geq$

$$
\frac{(1-\varepsilon) n+2(\varepsilon-1)\left[\sum_{1 \leq i<j \leq n}\left[\text { g.c.d. }\left(d_{i}, d_{j}\right)\right]^{2}\right]}{1-\varepsilon}
$$

$=n-2\left[\sum_{1 \leq i<j \leq n}\left[\text { g.c.d. }\left(d_{i}, d_{j}\right)\right]^{2}\right] \geq n-2 m$.

$E E_{G C D}(G)=n-2 m$ if and only if all the G.C.D. degree eigen values are 0 that is $G \cong K_{1}$.

Theorem 3.4Assume that $G$ is a $(n, m)$-graphwhich is simple and connected.. Then $E E_{G C D}(G) \leq \mathrm{e}\left[(\mathrm{n}-1)+\mathrm{e}^{E_{G C D}(G)}\right]$ and $E E_{G C D}(G)=\mathrm{e}\left[(\mathrm{n}-1)+\mathrm{e}^{E_{G C D}(G)}\right]$ if and only if $G \cong K_{1}$.

Proof:

$$
\begin{aligned}
& \text { Now } E E_{G C D}(G)=e \sum_{\mathrm{i}=1}^{\mathrm{n}} \mathrm{e}^{\lambda_{\mathrm{i}}-1} \\
& =e \sum_{i=1}^{n} \sum_{t \geq 0} \frac{\left(\lambda_{i}-1\right)^{t}}{t !} \\
& =e\left[n+\sum_{i=1}^{n} \sum_{t \geq 0} \frac{\left(\lambda_{i}-1\right)^{t}}{t !}\right] \\
& \leq e\left[n+\sum_{i=1}^{n} \sum_{t \geq 0} \frac{\left(\left|\lambda_{i}-1\right|\right)^{t}}{t !}\right] \\
& \leq e\left[n+\sum_{t \geq 0} \frac{\left(\sum_{i=1}^{n}\left|\lambda_{i}-1\right|\right)^{t}}{t !}\right] \\
& \leq e\left[n-1+\sum_{t \geq 0} \frac{\left(\sum_{i=1}^{n}\left|\lambda_{i}\right|\right)^{t}}{t !}\right] \\
& =e\left[n-1+e^{E_{G C D}(G)}\right]
\end{aligned}
$$

$E E_{G C D}(G)=\mathrm{e}\left[(\mathrm{n}-1)+\mathrm{e}^{E_{G C D}(G)}\right]$ if and only if all the G.C.D. degree eigen values are 0 that is $G \cong K_{1}$.

Theorem 3.5Assume that $G$ is a $(n, m)$-graphwhich is simple and connected. Then

Published By:

Blue Eyes Intelligence Engineering \& Sciences Publication

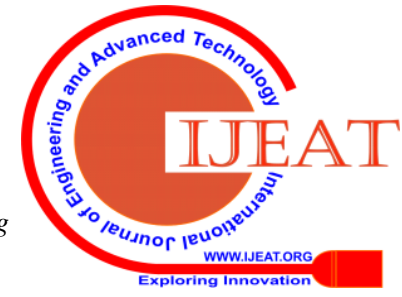




$$
\begin{gathered}
E E_{G C D}(G) \geq n+ \\
m+\frac{1}{2 e}\left[e^{2}-2 e-1\right] N_{3} \\
+\frac{1}{2 e}\left[e^{2}-3 e-1\right] N_{4}
\end{gathered}
$$

Proof:

$$
\begin{aligned}
& \text { Now, } E E_{G C D}(G)=N_{0}+N_{1}+N_{2}+\sum_{\mathrm{t} \geq 3} \frac{N_{t}}{\mathrm{t} !} \\
& \geq n+0+\frac{2}{2 !}\left[\sum_{1 \leq i<j \leq n}\left[\text { g.c.d. }\left(d_{i}, d_{j}\right)\right]^{2}\right]+\sum_{\mathrm{t} \geq 3} \frac{N_{t}}{\mathrm{t} !} \\
& \geq n+m+\sum_{\mathrm{t} \geq 2} \frac{N_{2 t-1}}{(2 \mathrm{t}-1) !}+\sum_{\mathrm{t} \geq 2} \frac{N_{2 t}}{(2 \mathrm{t}) !} \\
& \geq n+m+N_{3} \sum_{\mathrm{t} \geq 2} \frac{1}{(2 \mathrm{t}-1) !}+N_{4} \sum_{\mathrm{t} \geq 2} \frac{1}{(2 \mathrm{t}) !} \\
& =n+m+N_{3}\left(\frac{e-e^{-1}}{2}-1\right)+N_{4}\left(\frac{e+e^{-1}}{2}-1-\frac{1}{2 !}\right) \\
& =n+m+\frac{1}{2 e}\left[e^{2}-2 e-1\right] N_{3}+\frac{1}{2 e}\left[e^{2}-3 e-1\right] N_{4}
\end{aligned}
$$

\section{REFERENCES}

1. S.Alikhani, N.Ghanbari, More on Energy and Randic Energy of Specific Graphs, Journal of Mathematical extension, Vol.9, No.3(2015), 73-85.

2. R.Balakrishnan, The Energy of a graph, Linear Algebra Appl,3 87(2004), 284-295.

3. ChandrasekarAdiga and Smitha, On Maximum degree Energy of a Graph, IntJ.Contemp. Math.Sciences, Vol.4,2009, no 8, 285-396.

4. D.Cvetkovic, M.Doob and H.Sachs, Spectra of Graphs, Theory and Application, AcadamicPress,New York, 1980.

5. A.DilekGungor and A.SinanCevik, On the Harary Energy and Harary Estrada Index of a Graph, Match Commun. Math. Comput. Chem.,64(2010), 281-296.

6. I.Gutman, The Energy of a Graph, Ber. Math. Stat. Sekt. Forschungszent Graz, 103, (1978), 1-22.

7. G.Indulal, Sharp Bounds on the Distance Spectral Radius and Distance energy, Linear Algebra and its Applications 430, (2009), 106113.

8. R.S.Ramkumar and K.Nagarajan, Bounds for the Greatest Common Divisor Degree Energy of Graphs, Proceedings on International Conference on Recent Trends in Applied Mathematics, Jan 2017, 68-74.

9. R.S.Ramkumar and K.Nagarajan, Bounds on Greatest Common Divisor Degree Spectral Radius and Greatest Common Divisor Degree Energy of Graphs, Journal of Applied Science and Computations, Vol.5, No 11, Nov 2018,pp 1348-1353.

10. R.S.Ramkumar and K. Nagarajan, Greatest Common Divisor Degree Energy of Graphs, International Journal of Mathematical Sciences and Engineering Applications,Vol.11, No II, Aug 2017, pp 163-171.

11. R.S.Ramkumar and K.Nagarajan, Greatest Common Divisor Degree Estrada Index of Graphs, ICDCM 2019, Communicated.

\section{AUTHORS PROFILE}

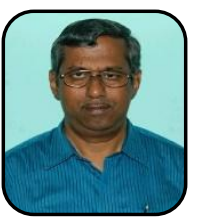

Dr. K. Nagarajan is working as a Professor of mathematics at Kalasalingamuniversity, Krishnankoil $\mathrm{He}$ was working as Head and AssociateProfessorof Department of mathematics at Sri S.Ramasamy Naidu Memorial College, Sattur. He has guided 38 M.Phil. Scholars and $4 \mathrm{Ph} . \mathrm{D}$. Scholars. He is currently guiding 5 Ph.D. Scholars. Having published 11 research papers in national level journals and 40 research papers in international level journals.He has completed Minor Research Project sponsored by UGC.He has 9 years experience in research and his area of interests in research is graph labelling, domination of graphs, distances in graphs and energy of graphs.

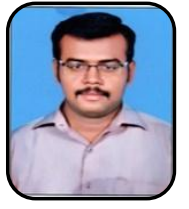

R. S. Ramkumar is working as Assistant professor of department of mathematics at Sri S.Ramasamy Naidu Memorial College, Sattur. He is pursuing his $\mathrm{Ph} . \mathrm{D}$. in Spectral graph theory under the guidance Dr.K.Nagarajan. He has completed his UG and PG degree at A.J.College, Sivakasi and M.Phil. Degree at Sri S.Ramasamy Naidu Memorial College, Sattur. His research work is concerned with the Energy of Graphs in the spectral graph theory.
Published By:

Blue Eyes Intelligence Engineering 